\title{
Emotion-Aware Education and Research Systems
}

\author{
Vladimir Jotsov \\ State University for Library Studies and Information \\ Technologies, Sofia, Bulgaria
}

jotsov@ieee.org

\begin{abstract}
An emotion-aware method KALEIDOSCOPE is presented in the paper. It consists of a visualization approach, a method for information transfer-by-sense and applied approaches to maintain a natural-style dialog. The combination is purposed to provoke and keep positive emotions in the user and to maintain his interest in the problem. The main goal of this paper is to show how different machine-done visualized patterns lead to student's perceptions that can't be represented or estimated by the machine and how this is helpful during the educational process. It is shown that dynamic information presentations are superior to static ones. Original evolving (dynamic) ontology applications have been introduced. It is shown that the creation and maintenance of such ontologies may be a rather complicated process. Essential principles of an educational process using KALEIDOSCOPE method are introduced. It is shown that high quality education systems are far away from monotonic presentation forms, and that they are rather close to contemporary research systems. Both education and research systems use a clear and thoroughly understandable dialog depending on user's know ledge level. The considered education system is an active part of the human-computer dia log process. Instead of trying to estimate the user's emotions we apply a set of logical data mining methods aiming at making the human-computed interaction emotionaware. The educational part of the system is domain independent. Aiming to show domain independent approaches, the examples are introduced from Number Theory to language expressions and nonclassical logic applications. Applications are presented in the domain of Number Theory and IT information security systems.
\end{abstract}

Keywords: Education Systems, Information Technologies, Emotion-Aware Systems, Emerging User Informing Technologies, Inte lligent System, Computational Discovery, Learning Formal Descriptions, Cognitive Psychology.

\section{Introduction}

An opinion is widely spread among lecturers and researchers in natural sciences and especially in mathematics that the usage of emotions in the professional context is by no means acceptable. It is accepted that there is no place for emotions in the Temple of Mathematics. Any too emotional

Material published as part of this publication, either on-line or in print, is copy righted by the Informing Science Institute. Permission to make digital or paper copy of part or all of these works for personal or classroom use is granted without fee provided that the copies are not made or distributed for profit or commercial advantage AND that copies 1) bear this notice in full and 2) give the full citation on the first page. It is permissible to abstract these works so long as cred it is given. To copy in all other cases or to republish or to post on a server or to redistribute to lists requires specific permission and payment of a fee. Contact Publisher@InformingScience.org to request redistribution permission. presentation may be rather subjective, it may hide details, it may induce negative reactions in the public, etc. It is shown in the paper that there exist different types of emotions hence the above assertions don't affect our research on emotional education in information technologies (IT). On the other hand, it is established that the reading speed for mathematical texts is much slower than 
for other texts (Eves, 1976). Some mathematical papers can be read much faster than others and sometimes it is due to emotional aspects. It is well known that creative processes in the scope are emotion-driven. This conclusion follows from the fact that processes for making formal proofs are long, the results and the difficulties can't be beforehand estimated, and almost nobody will take up with such a research without having anticipated the emotional and other rewards. Meanwhile when we omit the emotional factor in final publications, it makes the research look like a flower without roots. It becomes something nice but incomplete and not so vivid. This is the way the learning process becomes accelerated but it isn't of very high quality because the rote learning or learning by memorizing is frequently used. The rote learning is an antipode to the creative learning. Both types may be used in different situations but the creative element should be emphasized. The breakthrough in both IT- and mathematical education had been made by mid-XX century (Polya, 1963; The Erdos Project, 1985). Their books provoked strong emotional reactions and attracted the attention to Number Theory. Today there exist many different emotion-aware, affective computing and other relative systems applied in ambient intelligence etc. (ACII, 2005; Deransart, Hermenegildo, \& Maluszynski, 2000; MIT Media Lab, 2008; Pantic \& Rothkrantz, 2003). Most of them try to measure user's emotions while the latter author tries to provoke a creative research using positive user's state. Independently of them we elaborated a different set of methods aiming at making comfortable conditions for the user trying to learn new knowledge. We try to create such conditions that the trained person can manifest his/hers creative abilities. The above and below quoted research may be combined.

One of the identical features of a correct discourse between a group of researchers or between a teacher and a group of students is that every notion, every object, everything concerning the dia$\log$ should be clarified. On the other hand, if something is incompletely described or is explained in a wrong manner, it may lead to misunderstanding and consequently to wrong results. When the one side of the team members, namely the instructor, or the tutor, or the researcher, is a machine, the others receive many advantages: the dialog with the group may be split into many peer-topeer 'human-machine' dia logs. But the machine part is rather mechanical, still it cannot understand the sense of the notions it operates with. Aiming at reducing this drawback and improving the contemporary tutoring systems, the KALEIDOSCOPE method has been elaborated. Its nearest analogs are the so called brainstorming methods. When somebody uses a kaleidoscope, he enjoys the color patterns occasionally formed by the device. The kaleidoscope technology has been used in contemporary screensavers. Analogically the KALEIDOSCOPE method tries to find some regularity and/or to explain some aspects in such a way that provokes positive emotions in the user and an evident willingness for a creative investigation of the represented information. Here the computer system mechanically executes the mechanical part of the work, it does not estimate directly what is beautiful, what is interesting and so on. It swiftly catches repetitive patterns, chooses simple solutions because they are more effective than the complex ones and so on. Our experiments show, that when the educational (mechanical) part is represented in a correct way that is considered in the paper, it provokes an inexperienced user to further investigate the problems because they are presented in an interesting way to the user.

The main goal of this paper is to show how different machine-done visualized patterns lead to student's perceptions that can't be represented or estimated by the machine and how this is helpful during the educational process. The next section describes the basics of the KALEIDOSCOPE method, it is only one of the methods in our system. Also, for sake of brevity few other data mining methods are briefly described aiming to show different important features of the considered education system. For more information the reader may investigate the other quoted papers (Jotsov, 1999, 2002, 2008). After that the main features are discussed: visualization of the method, the transfer of a sense during the dialog, and approaches how to make the human-machine interaction productive. Applications, perspectives and the conclusion are represented in the final segment of the paper. 


\section{Application and Control of Emotions in Human-Machine Interactive Systems}

There exist many systems aiming to help users make creations like TechOptimizer of IMCorp, Engineering Center 'Creative Machine', etc. The quoted systems contain large bases of technological data but their human-machine interaction parts are not developed and maybe this is the main obstacle to the development of those systems. The considered KALEIDOSCOPE method is based on the following principles.

The machine should visualize the information in a manner to provoke the user's interest. The epoch of e-learning systems containing a bundle of lecture texts should disappear soon. The monotonic reading of books and web lectures leads to a poor and descending average quality of student's knowledge. The computer must be a more active part in the process of education.

- Comfortable, creative conditions for the user should be applied. Somebody may reply that education systems should be different from the research systems. The answer to this question is given below: the good educational process forces students to apply creatively what they have learned. Rote learning isn't the only alternative in education systems.

- The machine part of the educational process is by no means something extraordinary. It does not measure uncertain notions like beautiful, clear, innovative, etc. It executes its mechanical part and then represents it to the user in a 'provocative' manner as it is shown below.

- Everything is important during the visualization of the results. The educational process must be comfortable. Even the onscreen colors should represent a comfortable combination. Many of the above mentioned issues have been briefly described they cannot be introduced here, and will be reported later.

- Personalization is the key point of the system. The emotional contact could not be effective without memorizing the previous student activities.

- Different statistical methods are applied to measure the user's behavior. The classic statistical approach is shown in Figure 1A where the user's activity is statistically measured. In addition to this we apply different logical methods which substitute, when possible, probabilistic values during an evolutionary computation process. It is well known that statistical data mining methods do not effectively apply to logical data mining. Both logical and statistical data mining methods are combined in the proposed system as it is shown in Figure 1B. In such a way we successfully make formal proofs out of weak heuristic hypotheses (Jotsov, 1999).

- The sense in the educational process is transferred via ontologies. Evolving (dynamic) ontologies are more effective than static ones, but their construction and maintenance is much more complicated. We use static variants wherever possible.

- The following new forms of ontology applications are shown below.

- The system is rather transparent and it contains special dialog maintenance features. 


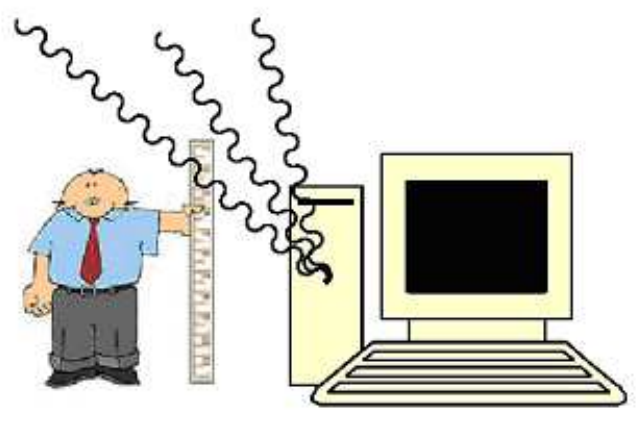

A.

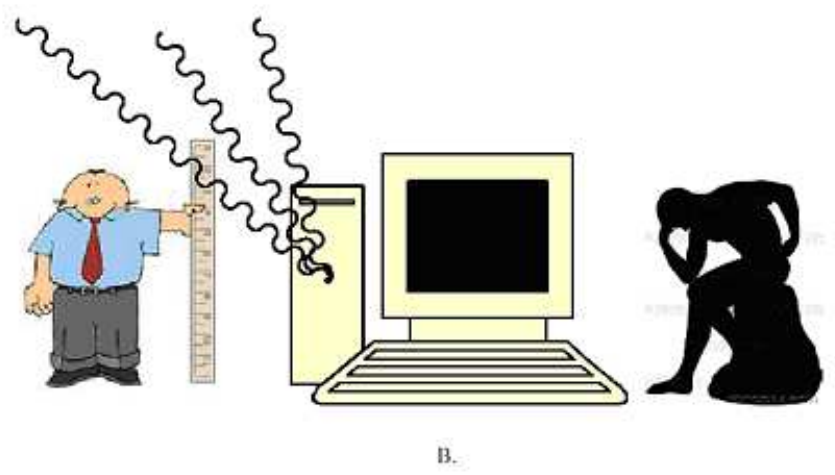

Figure 1: The combination of estimation me thods

The presentation must be clear. General notions must be applied, the most difficult parts must be isolated in a particular daemon-type section the level of the dialog should depend on each student's results and data.

The above quoted principles provoke positive emotions in the user and they reinforce both experienced and non-experienced students to further research the theme.

- It is shown in Figure 2 that many problems in IT and mathematics seem to be deceptively simple. Say, some simply formulated conjectures became $400+$ pages theorems. If the user is at point $\mathrm{A}$, the problem with a solution in point $\mathrm{B}$ has been formulated, and the user can't fly, then the resolution process may be much longer than the anticipated one. Sometimes it is not at all resolvable with contemporary methods: say, there is a precipice between A and B. For this reason the process of solving such problems is long and evolutionary and principally it is hard to resolve without an emotional contact between the program and the user. The evolutionary part of this research is represented in (Jotsov, 2002).

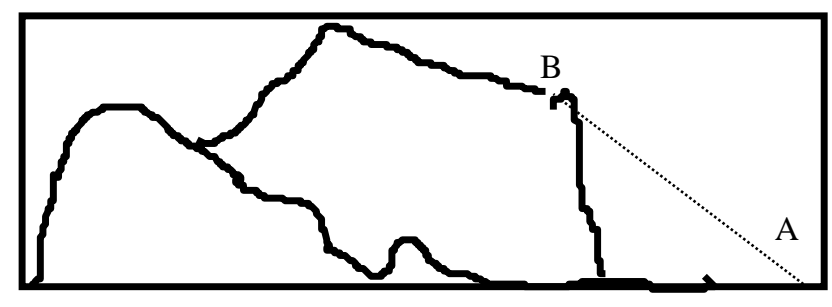

Figure 2: A goal and its solution

The main advantage of the below considered methods is revealed in the follow ing section. 


\section{Visualization}

We don't try to write a new theory of visualization for human-machine interactive systems. We shall provide operative examples of those applications that lead to successful results instead. Although the main topic of this paper is the IT education, the examples here are from Number Theory due to the following reasons. Mathematics is the basis for most IT education fields, and mathematical examples are free of heuristics, representing more powerful, short, and clear data. An example of operation with number concepts is depicted below.

A set of eight arithmetic progressions is represented in Figure 3. It is made by sieving out prime numbers 2, 3 and 5 and the ir composites from the set of all natural numbers, the positive integers. In Figure 4 the machine shows a multiplication cycle for number 11, this means 11 times all the consecutive numbers from Figure 3, introduced line by line as it follows: 11 (times 1), 77 (times 7), 121 (times 11), 143 (times 13), 187 (times 17), 209 (times 19), 253 (times 23), and 319 (times 29). The pattern is named a cycle, because when 11 has been multiplied by the consequence of the numbers from line 2 in Figure 3, then the pattern is the same but it is shifted with 314 (11 times 31$)$. The machine easily reveals such simple regularities.

\begin{tabular}{|llllllll|}
\hline 1 & 7 & 11 & 13 & 17 & 19 & 23 & 29 \\
31 & 37 & 41 & 43 & 47 & 49 & 53 & 59 \\
61 & 67 & 71 & 73 & 77 & 79 & 83 & 89 \\
91 & 97 & 101 & 103 & 107 & 109 & 113 & 119 \\
121 & 127 & 131 & 133 & 137 & 139 & 143 & 149 \\
151 & 157 & 161 & 163 & 167 & 169 & 173 & 179 \\
181 & 187 & 191 & 193 & 197 & 199 & 203 & 209 \\
211 & 217 & 221 & 223 & 227 & 229 & 233 & 239 \\
241 & 247 & 251 & 253 & 257 & 259 & 263 & 269 \\
271 & 277 & 281 & 283 & 287 & 289 & 293 & 299 \\
301 & 307 & 311 & 313 & 317 & 319 & 323 & 329 \\
331 & 337 & 341 & 343 & 347 & 349 & 353 & 359 \\
361 & 367 & 371 & 373 & 377 & 379 & 383 & 389 \\
391 & 397 & 401 & 403 & 407 & 409 & 413 & 419 \\
421 & 427 & 431 & 433 & 437 & 439 & 443 & 449 \\
\hline
\end{tabular}

Figure 3: The eight progressions containing all primes $>5$

In Figure 5 another multiplication cycle for number 19 is shown. It is seen evidently that $11+19=30$ and that 30 is the progression step. The machine uses rather simple algorithms to reveal that cycle 19 is the same as cycle 11 , stretched and rotated to 180 degrees. The user easily reveals this fact. Is the complementary character of patterns from Figure 4 and Figure 5 arbitrary? No, the cycles for 7 and 23, 31 and 59 etc. show the same behavior, the cycle for 41 is the same as for 11 but it is stretched, and it returns to the same row after 1230 (41 times 30) instead of 330 in cycle 11.

And why the progression step is equal to a multiplication of all the sieved out prime numbers? Why each pattern has a center of symmetry? Why just these 8 progressions have been used? The answer to the latter question is: because if less prime numbers are sieved out, then the multiplication cycles become absolutely identical, and the difference between them is that one of them is more stretched than another: no rotations, etc.

The fragments from Figure 3 up to Figure 5 show more data for further comparisons. If more prime numbers are sieved out, the figures become too complicated. Maybe they will be fruitful in further investigations. 


\begin{tabular}{|llllllll|}
\hline 1 & 7 & 1 & 13 & 17 & 19 & 23 & 29 \\
31 & 37 & 41 & $\mathbf{4 3}$ & 47 & 49 & 53 & 59 \\
61 & 67 & 71 & 73 & 77 & 79 & 83 & 89 \\
91 & 97 & 101 & 103 & 107 & 109 & 113 & 119 \\
121 & 127 & 131 & 133 & 137 & 139 & 143 & 149 \\
151 & 157 & 161 & 163 & 167 & 169 & 173 & 179 \\
181 & 187 & 191 & 193 & 197 & 199 & 203 & 209 \\
211 & 217 & 221 & 223 & 227 & 229 & 233 & 239 \\
241 & 247 & 251 & 253 & 257 & 259 & 263 & 269 \\
271 & 277 & 281 & 283 & 287 & 289 & 293 & 299 \\
301 & 307 & 311 & 313 & 317 & 319 & 323 & 329 \\
331 & 337 & 341 & 343 & 347 & 349 & 353 & 359 \\
361 & 367 & 371 & 373 & 377 & 379 & 383 & 389 \\
391 & 397 & 401 & 403 & 407 & 409 & 413 & 419 \\
421 & 427 & 431 & 433 & 437 & 439 & 443 & 449 \\
& & & & & & & \\
\hline
\end{tabular}

Figure 4: Multiplication cycle for 11

\begin{tabular}{|llllllll|}
\hline 1 & 7 & 11 & 13 & 17 & 19 & 23 & 29 \\
31 & 37 & 41 & 43 & 47 & 49 & 53 & 59 \\
61 & 67 & 71 & 73 & 77 & 79 & 83 & 89 \\
91 & 97 & 101 & 103 & 107 & 199 & 113 & 119 \\
121 & 127 & 131 & 133 & 137 & 139 & 143 & 449 \\
151 & 157 & 161 & 163 & 167 & 169 & 173 & 179 \\
181 & 187 & 191 & 193 & 197 & 199 & 203 & 209 \\
211 & 217 & 221 & 223 & 227 & 229 & 233 & 239 \\
241 & 247 & 251 & 253 & 257 & 259 & 263 & 269 \\
271 & 277 & 281 & 283 & 287 & 289 & 293 & 299 \\
301 & 307 & 311 & 313 & 317 & 319 & 323 & 329 \\
331 & 337 & 341 & 343 & 347 & 349 & 353 & 359 \\
361 & 367 & 371 & 373 & 377 & 379 & 383 & 389 \\
391 & 397 & 401 & 403 & 407 & 409 & 413 & 419 \\
421 & 427 & 431 & 433 & 437 & 439 & 443 & 449 \\
451 & 457 & 461 & 463 & 467 & 469 & 473 & 479 \\
481 & 487 & 491 & 493 & 497 & 499 & 503 & 509 \\
511 & 517 & 521 & 523 & 527 & 529 & 533 & 539 \\
& & & & & & & \\
\hline
\end{tabular}

Figure 5: Multiplication cycle for 19

Our experiments show that even inexperienced students may come to serious mathematical theorems and to new hypotheses after the investigation of pictures that are similar to the presented ones. The purpose of the figures and the explanations of the above sample with arithmetic progressions is not an explanation of mathematical regularities but to show that visualization of certain mathematical results is interesting by itself. Otherwise when the user looks at the lines of figures 4 and 5 then in the majority of cases he/she will be interested in the pattern and he/she will ask the above stated questions supportive the further research. But when he/she sees that fragments are repeated then this will deepen the interest in the problematic field which leads to interesting mathematical regularities. At that the machine does not estimate the beauty or the usefulness of the fragments. The machine just arranges data in such way that it provokes the necessary emotions in the user. In this way necessary conditions are created for training and research respectively.

Imagine that in this fragment we try to measure user's emotions to manage in a better way the problem solving process. In this way we can only fill the system with heuristics but we cannot improve the human-machine interaction because frequently human emotions are deceptive, e.g. one smiles when he/she feels very badly. The difference from direct emotion-aware methods is in our proposition to implicit effect the user. Our proposition is untraditional but it is possesses the basic properties of other emotion-aware methods.

Other particular visualization technologies are discussed in the next section. 


\section{Methods for Transfer of Sense during the Education}

Actually we used in the previous section different methods to form particular ontologies (or shared conceptualizations) during the human-computer dialog. Ontologies are the base knowledge used in IT (Russell \& Norvig, 2003). Below our approach is represented to manifest the idea of know ledge management in the IT educational process.

\section{Ontologies for Education}

As in most similar research we use the Protégé ontology editor (Protégé ,2008) to form essential static ontologies of a vocabulary type like the shown ones in examples in from Figure 6 and Figure 7.

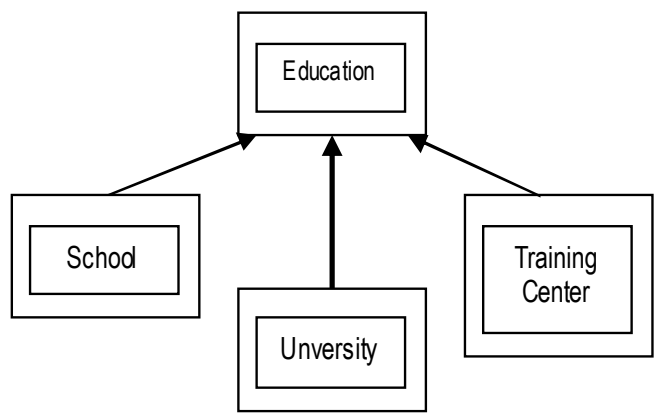

Figure 6: Education ontology.

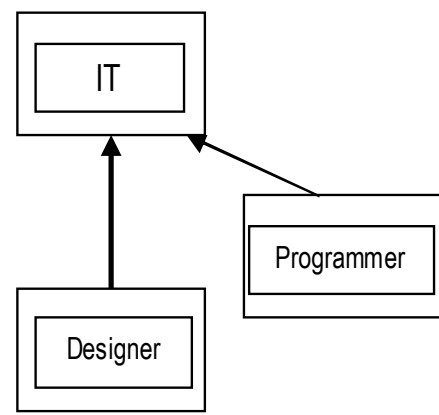

Figure 7: IT ontology.

They form complex ontologies that are similar to the IT example depicted in Figure 8. These simple forms of knowledge are used to reveal the type of relations between different used notions. Using the taxonomy ontology from Figure 6, the machine will 'realize' that the user asks something about the education or lectures when he enters the keyword 'university'.

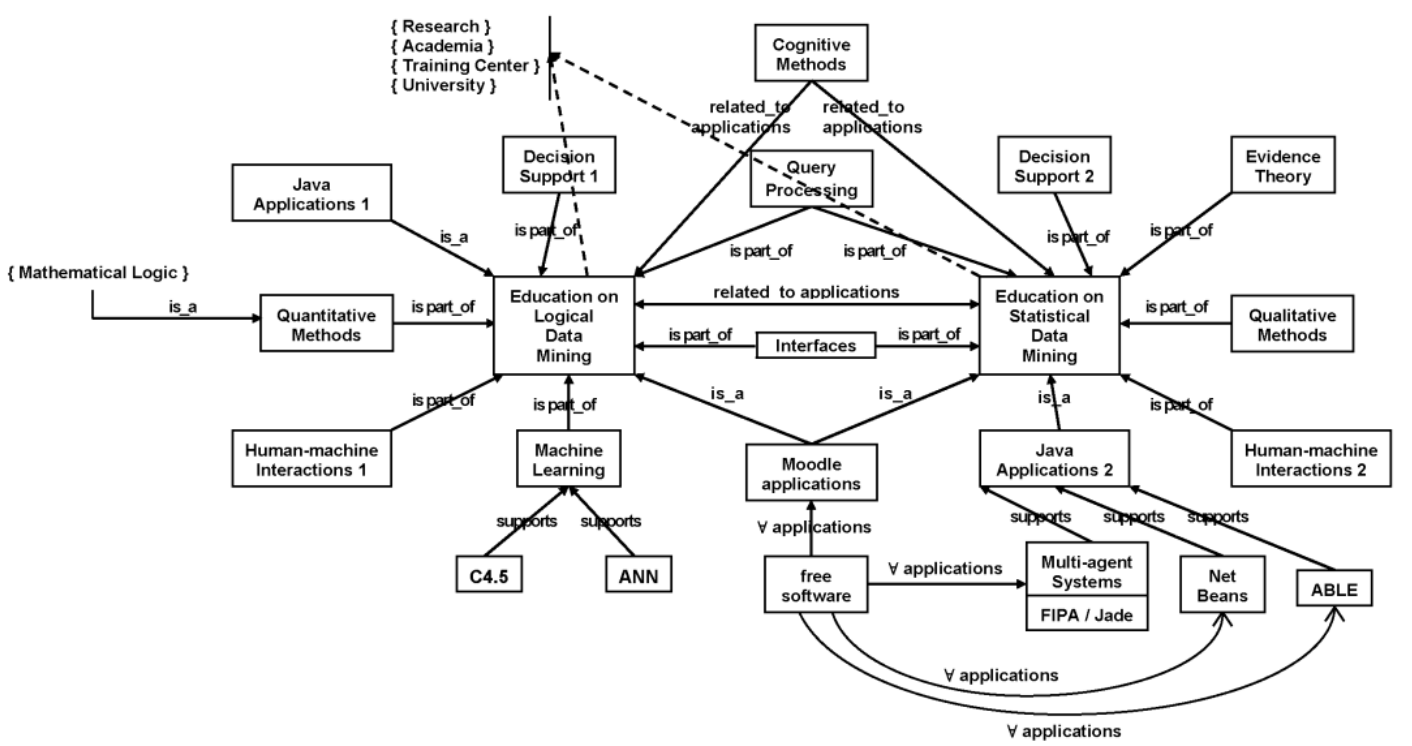

Figure 8: An example of a data mining ontology 


\section{Visual Patterns and Ontologies}

Not all ontologies are graph structures. We frequently use pictures like Figure 9 instead. Some users will see in the picture interesting details about taking on/off the water. Other ones will re lax looking at the beautiful bird. There is also a lot of other information concerning the figure.

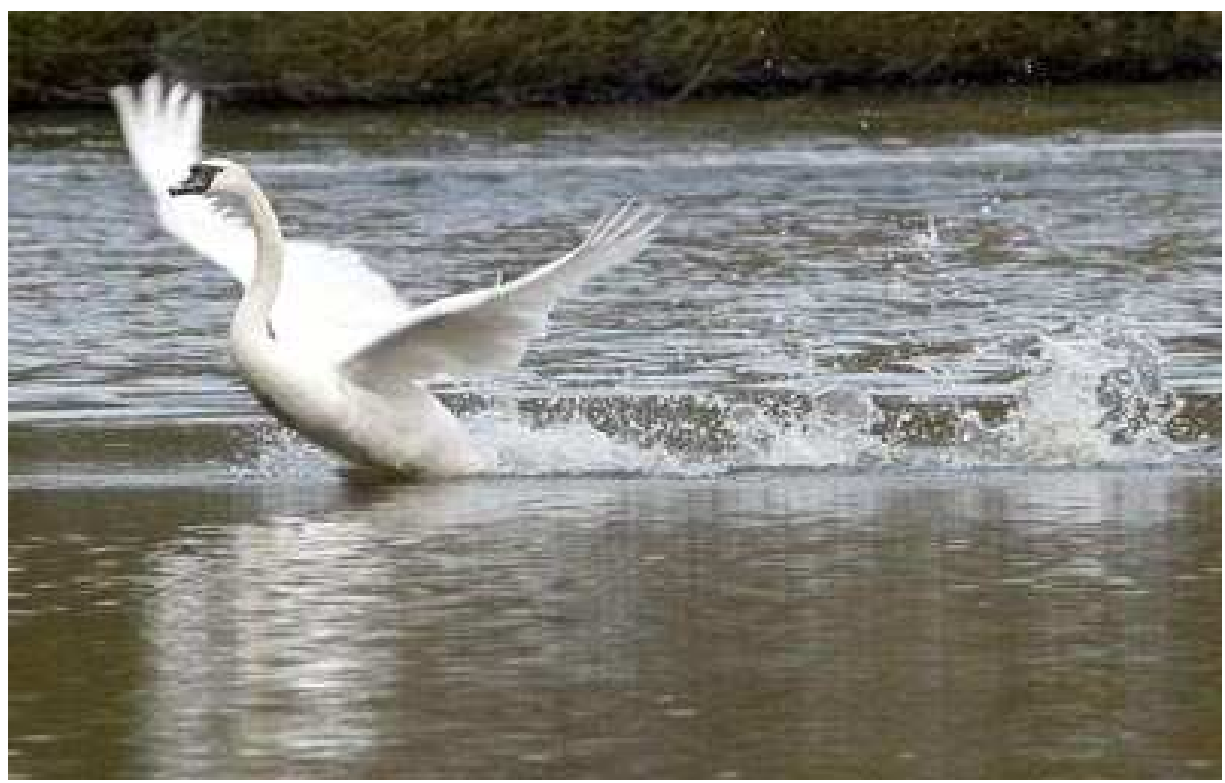

Figure 9: Information for analysis by sense from water birds

Definitely the picture provokes different emotions and just similar emotions remain in the memory afterwards. Again, here the machine doesn't formulate and maintain emotions, they are provoked by an appropriate picture. To orientate the training process in the right direction we use various forms of metaknow ledge. If the research includes taking off the water then the motto of the picture will be 'see how birds take off the water'. Doubtlessly it raises much more emotions than standard text descriptions. Cited metaknowledge is contained in ontologies accompanying the picture. Every picture could be transformed to an ontology and as it is shown above the ontology can be represented as a picture (see Figure 4 or 5). The ontology from Figure 9 is a static one, but it will switch to the dynamic variant when a movie is shown instead of it. If the picture shows a key moment from the flight then in an educational aspect it may replace the whole movie. Surely the representation of a key moment from some significant event raises no less but even more emotions than the event as a whole. It will be shown below that the right combination of ontologies and the visual contents leads to a qualitatively better education. We shall discuss original ontology variants in the next subsection.

\section{Explanation Based Ontologies}

To make the dialog natural and the education effective, we elaborated and applied the following forms of knowledge representation, management and explanation.

Dynamic ontologies in other contemporary systems are used to append, update, mainta in or produce the ontology bases. In Figure 10 a different way is shown. Its motto is 'bind the unknown to known' and it already brought effective applications in IT education. Say, let the system explains section integrals from mathematical analys is and the student shows some misunderstanding. If the system compares the student's knowledge in differential equations and reveals high results, then the explanations 'to integrate is similar and opposite to differentiate' will be helpful. This is a natural way of communication and a basis for positive emotions. 
If the goal $\boldsymbol{G}$ from Figure10 is different: 'what is $G$ ?', then the system has the following ways to resolve the problem.

- The constraints $\mathrm{V}$ form an area that includes $G$. Say, the education should be effective, emerging technologies should be used, the lecturers should be distinguished, so $G$ is: 'take the most expensive hardware' or an analogical choice.

The constraints V must be replaced by ontologies if possible. Say, we don't know the solution to the problem, but it be longs to an ontology 'universities in NYC'. Such ontology replaces a complicated system of constraints, many of them may be nonlinear or fuzzy and such a system may have no acceptable solutions. In any case our ontology application is much more effective than the classical constraint satisfaction methods.

The intersections A, C, and E in Figure 10 are just 'binding the unknown to known'. We may explain this principle by an analogy to a multi-dimensional crossword or to a sudoku. Even if their application doesn't resolve the goal $G$, then it reveals parts of the solution and it facilitates the explanations.

- In Figure 10, the black lines E and B bring no solutions to the problem $G$. They show binding intersections close to the problem instead, they 'fix up the goal' and they are applied analogically to A, C or E.

- Let us suppose that line E dynamically moves and it is anticipated that soon it will intersect with $G$. In this case a much more emotional text 'the things tend to ...' may be shifted by few figures like Figure 10. In the same way other natural conversation texts may be formalized or elicited from Figure 10:

'it is neither... nor... but it is pretty close to...',

'if we turn the tables, the result will be different...',

'the solution is not A or B but if $\mathrm{X}$ and $\mathrm{Y}$ then it is $\mathrm{C}$ ', etc.
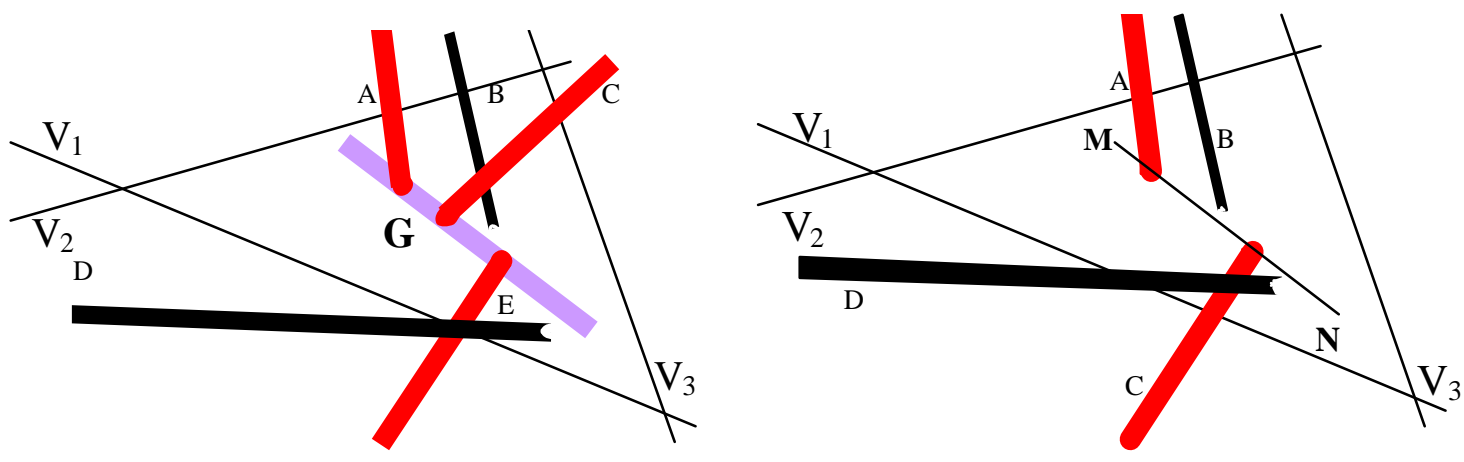

Figure 10: The area for resolution of the goal.

Figure 11: Reveal the causal co nnection M-N.

Another application of the same proposals is shown in Figure 11. The same set of constraints and binding elements is applied to show the causal connection between $\mathrm{M}$ and $\mathrm{N}$. As a result the user may be surprised by new conclusions, say 'strong computer science methods may have successful applications in abstract mathematics'. Any surprising information may be a driving factor to creative decisions. 


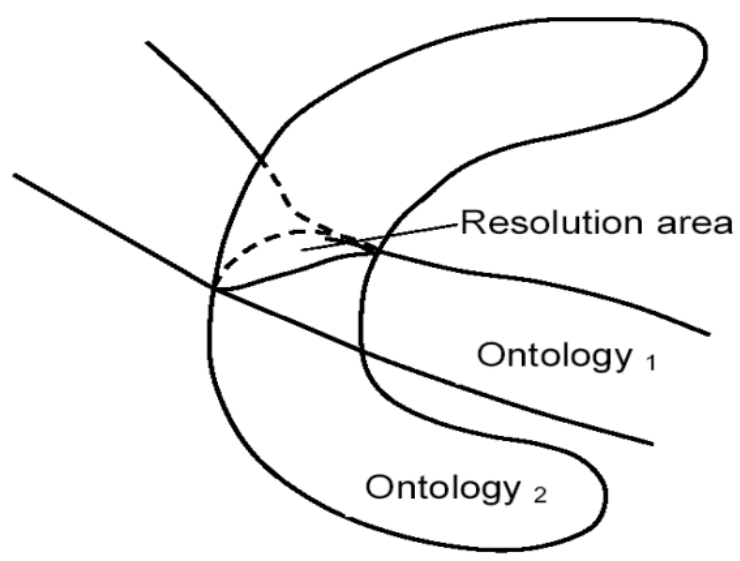

Figure 12: An intersection of ontologies forms a fuzzy constraint area

It is shown in Figure 12 that the intersection of different ontologies makes the problem solving easy. Let the goal lies in the intersection of ontology 1 and ontology 2 . Then the educational process is not only simplified but it is also more natural and understandable by the students. For example, we applied the following solution to the goal 'how to make student know ledge in IT lectures better':

- we made the presentation simple and emotional;

- we showed recent results, know-how and a strategic view about the field;

- let the complex and technical methods be learned by students at home or in libraries, preferably using e-learning tools;

- we implied a strict control of the auditory and we made changes in time.

All this principles are known to everybody in the domain but if we try to formulate it using classical constraint satisfaction methods, we'll fail. Here ontologies must be used, and the result belongs to the ir intersection. Also it is here that the natural and emotional ways coincide.

\section{Evolution of Ontologies}

Our research shows that using static ontologies is not enough in the IT educational process. Applying evolving (dynamic) ontologies is a more natural way of education. Most IT fields are rapidly changing. The sense of many notions undergoes dynamical shifting. The paradigms also may change their direction. Say, few years ago the mainstream of hardware evolution was a higher chip frequency. Today it is multicore, tomorrow another idea will dominate. The education know ledge should evolve, and showing this evolution will be very useful to IT students. The evolutionary process is depicted in Figure 13. The old direction is depicted by the blue ' $\mathrm{f}$ '. It is analogous to the fitness function in many traditional evolutionary methods. The contemporary direction is the red line. The set of constraints is depicted in the form of a funnel. Students better understand the basic notions when the changes in them are shown in an evolutionary way, but not via the 'revolutionary way'. It may be shown that the development direction is rather loose in the beginning in point B but in the end of the period it is very narrow in point $\mathrm{E}$. In any case, the usage of general factors is similar to the gravity in a funnel: it is rather weak compared to other forces but it is the one that humans can hardly escape from. For sake of brevity, analogies are frequently used to explain the basic features of the considered research. The usage of analogies makes our explanations more emotional, less complex, and more understandable. The process of 
the knowledge management in the considered education systems should be evolutionary as depicted in Figure 14. The applications are based on our previous work (Jotsov, 2002).

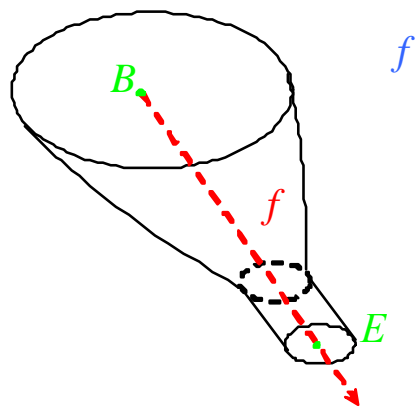

Figure 13: Constraint area in the FUNNEL.

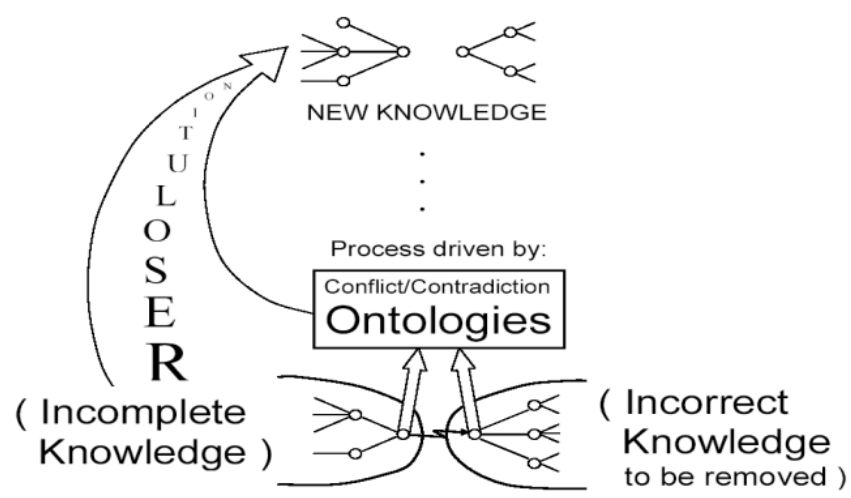

Figure 14: Development of ontologies

In the end of this section we shall discuss the question how to process incomplete or incorrect ontologies. Almost every part of knowledge in the education system is either imcomplete or incorrect. In education systems this leads to incorrect explanations, thus diminishing the trust in elearning systems (or lecturers) and it quits lots of poss ibilities for an effective learning. To cope with this problem, we created a tool for identification and resolution of conflicts in ontologies or any other knowledge (Jotsov, 2008). Ontologies in our systems pass through contradiction resolution or they may be artificially put in another field to reveal its strong and weak aspects. After resolving the contradictions, the quality of ontologies improves significantly. Furthermore, we elaborated a machine learning method which is based on constant comparisons between pairs of know ledge aiming at revealing and resolution of contradictions. This machine learning doesn't depend on a teacher, and the knowledge base is gradually self-improved. Only several complicated cases of contradictions are shown to experts. Most of the cases are resolved automatically. In such a way the students' trust to the education system is not provoked by tutoring errors and the applied conditions are creative.

The visualization of the proposed new learning scheme through contradiction resolution (Jotsov, 2008) makes the education process of IT students much more effective and emotionally saturated. The reason is that the automatic or semiautomatic process of localizing and correcting errors is very natural, instructive and it offers easy-to-remember knowledge to creatively cope with difficult situations. 


\section{Making the Dialog more Informative}

We applied student contests and other forms for active user participations. At the lower level a dialog-inspiring positive emotion is applied. Say, 'your results are among the best 10\%' etc. The latter is a wide-spread example but here it aims to illustrate the cognitive approach with the motto 'try to find good things about the student and trying to establish emotional contact to him'.

Usually the information in the education system is created not by just one but by many experts. To avoid a misunderstanding due to a poor knowledge fusion, we apply the following metaknowledge.

- Who is the knowledge creator.

- When the knowledge has been created.

- Why, when, and who made the changes.

- What are the changes.

- What is the status of the knowledge (draft, incomplete, ready-to-use).

- Students can grade any piece of lecture at any time.

Many of the described procedures rely on the usage of different models or ontologies in addition to the domain know ledge applied as metaknowledge forms. In a knowledge-poor environment human-machine interactions have a great role, and the metaknowledge helps make the dialog more effective and less boring to the human. The dialog forms are divided in 5 categories from $1=$ 'informative' to $5=$ 'silent' system. Attention: from the user's choice it is possible to judge about his/hers emotional state: e.g. if he/she is not in a good mood then it will choose 1 . This is the only entry in our research where it is possible to estimate the emotional state of the student. As it is shown above, in all other cases actions are undertaken for establishing an emotional contact, not to estimate user's actions. Knowledge and metaknow ledge fusion must always be documented: where the knowledge comes from, etc. This is our main principle: every piece of knowledge is useful and if the system is well organized, it will help us resolve some unexpected situations.

The above shown static ontologies are appended in the following way. New relations are introduced, say 'BASE', the spirits is the basic part of every alcohol beverages (Figure 15). Another relations shown in Figure 16 are 'origin', 'why', 'how', 'what for'. Say, the origin of the wine is Mediterranean, and for the wine it may be denoted 'unknown'. The latter 3 relations give more freedom to students. At every moment they may ask 'what is this explanation for why is this explanation', 'why did you use this notion here', 'how to explain this', etc. The system answer by now is realized in the simplest form, it is in the form of a prepared text. In future a reasoner agent will replace it. 


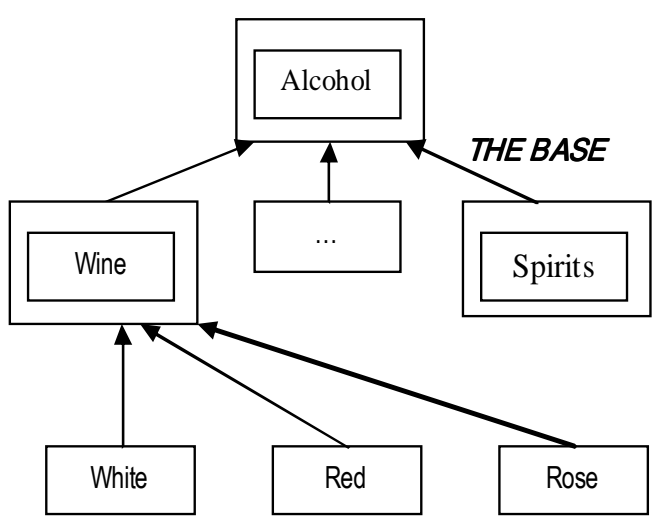

Figure 15: Ontology for alcohol beverages.

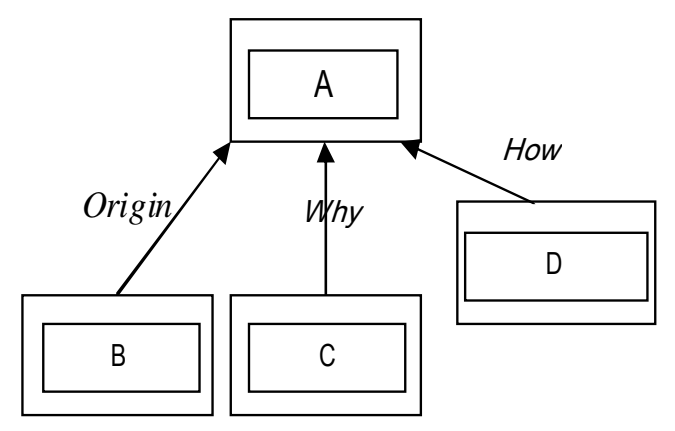

Figure 16: New semantic relations introduced by us.

We try to use a clear presentation style, we avoid wherever possible fuzzy, uncertain notions, we use generalizations to find clear words instead. Say, 'student', 'budget' instead of 'person with comparative ly low wages', 'unknown lump of money'.

At an application level we use more adjectives, and other means to provoke emotions in the user and to make the dialog natural. In any case the considered tools are not applied in the efficient way, and we are waiting for a dialog with professional psychologist auditory for further improvements.

\section{Applications}

It is a pity but when we speak about applications of the presented above methods we by necessity will move away from the educational and cognitive aspects of the research. We shall take this risk to illustrate briefly applied aspects of the considered research.

The presented system source codes are written in different languages: C++, VB and Java. All the ongoing research is done in Java because the multi-agent FIPA-based software is used. An application example for the security subsystem of the KALEIDOSCOPE method is shown below aiming at demonstration of new features of the well-known multiagent systems..

A fragment of the Java class Estimator is shown in Figure 17. An Estimator receives messages from agents capable to execute a chosen goal and is calculating their competence. The first agent or set of agents with amply high competence will receive the job. The competence of agents is a constantly changing value depending on the job type and the number of sweepstakes collected by an agent, say, the set of realized goals, etc.

Conflict situations occur in the module from Figure 17 when the agent can't execute its goal in an acceptable time or in other situations. Say, the agent's way to the goal is interrupted by a route for a number of other agents. The standard way frequently quoted in multiagent systems is 'step out' for all conflicting agents. Then another routes must be found, otherwise the same conflicts will repetitively occur. Meanwhile some goals may be lost. On the other hand, one or more of the considered above conflict-resolving methods solve the problem using the well known class Agent and methods like halt(); suspend(); activate(); getState(); move(Location where); ometimes clone(Location where, java.lang.String newName). Several libraries for class Agent are used. All of them comply with the FIPA standards. 


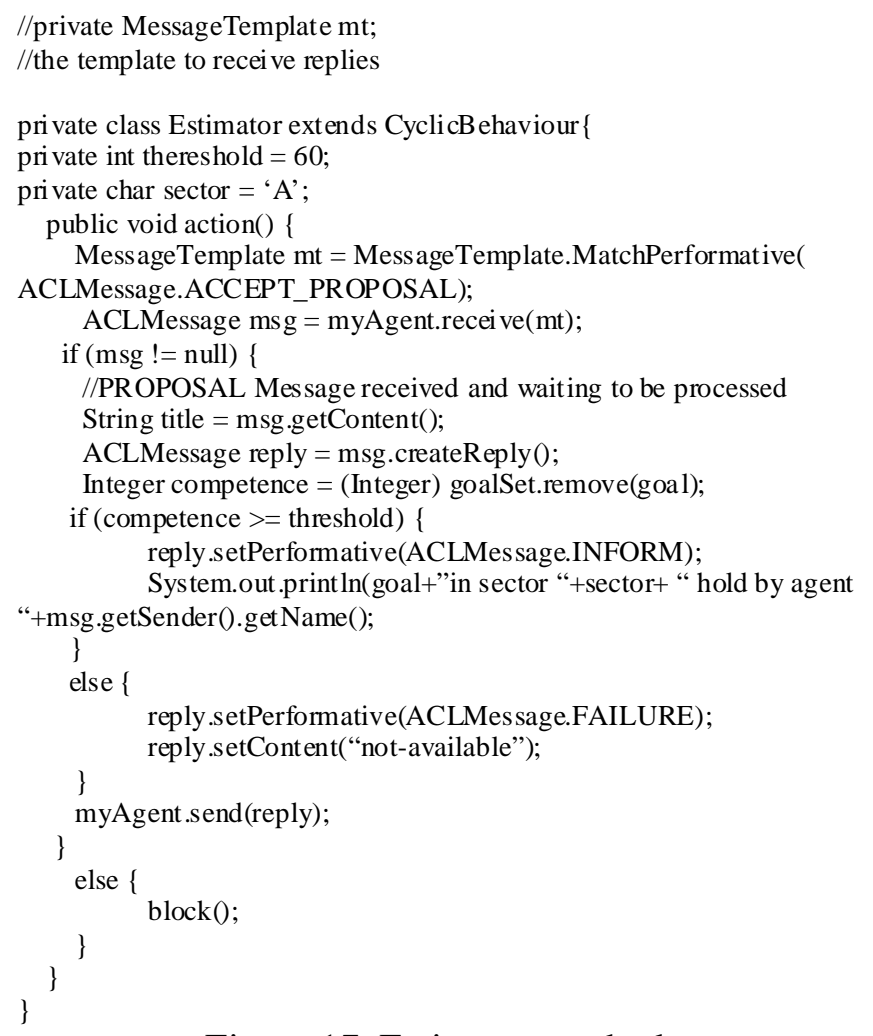

Figure 17: Estimator method.

Application examples for other methods are much more complex and cannot be described here. They show us that the considered methods are not so scholastic as it may seem at a first glance. Say, the method depicted in Figure 10 or Figure 12 and in Figure 14 could be effectively used to find intersections between different agent ontologies an so on.

We rely on non-symmetric reply to intruders with a motto surprise and win, on the usage of unknown codes in combination with well known methods, and on the automatic-reply high speed in some simple cases e.g. to halt the network connection when the attack is detected. If any part of the system is infected or changed aiming at reverse engineering or other goals, then the system will automatically erase itself and in some evident cracking cases a harmful reply will follow. The above presented models of users and environment(s) are used in the case.

In many applications it is necessary to apply fuzzy logic. This issue, and most of the applications' parts have not been considered in this paper, because its scope is to represent the core of the ongoing investigation and research. On the other hand, Figure 13 shows our or iginal realization of the fuzzy principle: we avoid a lot of hard-to-explain fuzzy notions via using ontologies and intersections between them. Many technical details are quoted in our references to this paper (Jotsov, 1999, 2008). The success of the presented application(s) is hidden in a rather simple realization of the presented methods. We tried to make complex applications using reasoning by analogy, machine learning or statistical data mining methods but in this case the complexity of the prototype system is greater than NP-hard.

The presented applications are purposed for realizations in the Moodle online education system (Moodle, 2008). 


\section{Conclusion}

People apply many different emotions when using education or other computer systems. We apply user interaction and personalization tools, but we do not try to measure user emotions. The present part of the investigation may be combined with affective computing applications to ambient intelligence, etc. The trial is to provoke and maintain positive emotions during the work with IT education systems. A set of original visualization methods aims to turn the user to his little, or maybe big, creations. The main idea of KALEIDOSCOPE is close to brainstorming methods or to some advanced computer games. Apart from the latter, we do not go to extremes: the user proceeds further only if he chooses the option, he can use standard queries or ask to repeat any part of the presentation at any time.

Ontologies are used instead of many fuzzy or uncertain notions in the educational module. Operations with ontologies are used instead of the nonlinear of fuzzy constraint satisfaction. The considered KALEIDOSCOPE method and its applications lead to a natural way for the dialog 'student-system' and it leads to a higher-education level and research results. Interactions of KALEIDOSCOPE with logical data mining methods FUNNEL and CROSSWORD are briefly described. More details on the system as a whole may be obtained from (Jotsov, 1999, 2002, 2008). Only the visualization part of the applications of FUNNEL and CROSSWORD has been considered in the paper, the other parts of the research may be found in the other quoted papers and in the ongoing research. It shows how the contemporary logical data mining methods help to establish emotion-aware contacts during the education process.

The ongoing research will proceed to develop group human-computer KALEIDOSCOPE methods, with applications of data mining methods, explanations by analogy, associations, comparisons, agents of a reasoner type, and some emerging technologies to IT education systems based on Moodle. The main obstacle is the high computational complexity of the perspective methods and applications.

\section{Acknowledgement}

This paper is granted by the project 'Dynamic Ontologies in Multi-agent Information and Control Systems'. Contract with the Bulgarian Ministry of Education and Science.

\section{References}

ACII. (2005). Proc. A ffective computing and intelligent interaction. International conference, Beijing, CHINA, 22/10/2005. Lecture Notes in Computer Science, 3784. Berlin: Springer Elsevier.

Deransart, P., Hermenegildo, M., \& Maluszynski, J. (Eds.). (2000) Analysis and visualization tools for constraint programming. Lecture Notes in Computer Science, 1870. Springer Elsevier.

The Erdos Project. (2008). Collected papers of Paul Erdös. Retrieved November 29, 2008, from http://www.renyi.hu/ p erdos/

Eves, H. (1976). An introduction to the history of mathematics. NY: Holt, Rinehart and Winston.

FIPA. (2008). Foundation for intelligent physical agents. Retrieved September 30, 2008, from www.fipa.org

Jotsov, V. (1999). On one approach in proof search and refinement. J. Artif. Intell., 2, 97-103.

Jotsov, V. (2002). Evolutionary parallels. Proceedings of the First International IEEE Symposium 'Intelligent Systems' (Vol. I). Varna, Bulgaria, September 10-12, 2002.

Jotsov, V. (2008). Novel intrusion prevention and detection systems. In R. Yager, V. Sgurev, \& V. Jotsov (Eds.), Proceedings of the $4^{\text {th }}$ International IEEE Conference on Intelligent Systems (Vol. II) Varna, Bulgaria, Septe mber 6-8, 2008. 
MIT Media Lab. (2008). Affective computing group. Retrieved August 15, 2008, from http://www.affect.media.mit.edu/projects.php

Moodle. (2008). Moodle is a course management system designed to help educators who want to create quality online courses. Retrieved Septe mber 30, 2008, from http://moodle.com/

Pantic, M., \& Rothkrantz, L. (2003). Toward an affect-sensitive multimodal human-computer interaction. Retrieved September 9, 2008, from $\underline{\mathrm{http}: / / \text { www.kbs.twi.tudelft.nl/People/Staff/M.Pantic/ProcIEEE- }}$ PanticRothkrantz.pdf

Polya, D. (1963). How to solve it. NJ: Princeton University Press.

Protégé. (2008). The Protégé ontology editor and knowledge acquisition system. Retrieved September 30 , 2008, from www.protege.stanford.edu

Russel, S., \& Norvig, P. (2003). Artificial intelligence: A modern approach (2nd ed.). NY: Prentice Hall.

Simonite, T. (2007). Emotion-aware teaching software tracks student attention. Retrieved November 29 , 2008, fro m http://www.news cientist.com/artic le/dn 10894-emotionaware-teaching-software-tracksstudent-attention.html

\section{Biography}

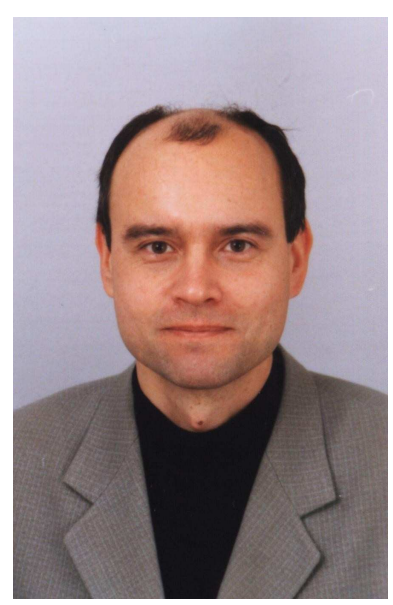

Vladimir S. Jotsov

Research, Educational and Scientific Experience: 20+ years. Author of 1 monograph book and approx. 90 scientif ic publications. Professor with broad scientific know ledge in the fields of: Artificial Intelligence, Applications to Information Security Systems, Evolution in Mathematical Proofs, Evolving Ontologies, Automation of Creative Processes, Inference by Analogy, Computational Discovery, HumanMachine Interaction, Cognitive Systems, Applications of Informatics to Number Theory, Knowledge Discovery and Data Mining, Decision Support Systems, Knowledge Management Systems, etc. Computer scientist/know ledge engineer, chair or coordinator of big scientific or international professional or work groups. Extensive teaching experience - tutor of 30+ undergraduate and postgarduate students.

Expe rience in software development: 24 years. First systems have been made as a student and simultaneously a researcher at National University KPI (USSR). Security applications in banks, education, research, etc.

Programmed in Java/J2EE, C/C++, JavaScript, Prolog, V isual Basic/Excel macros, Qbasic, Fortran, Pascal, P1/1, Assemblers/Macro Assemblers. Certified Java and SAS Base Programmer.

Systems/data bases used: SAS, SQL Server 2000/2005, Access, MUMPS (in VMS) and many other relational data bases.

Administrative experience: $15+$ years. Chair of research, technical or workgroups (up to 50 people). Head of Department Communications and Security, SULSIT-Statye University in Sofia. Was Head of Department IT in SULSIT-Sofia, Head of Computer Laboratory: 150+ PCs, security policy maker, etc.

Project experience: 20+ years. Expert in European Commiss ion (FP7), theme Security. Project coordinator, project leader or participant in approx. 20 national, European and US-projects. 